\title{
Restauração indireta Endocrown: Relato de caso clínico
}

\section{Indirect Endocrown restoration: Clinical case report}

Ana Luísa de Araújo Ávila1, Vitória Almeida Ramos², Sônia Maria Paiva Torres³.

\section{RESUMO}

Introdução: A reabilitação estética e funcional de dentes com extensa destruição coronária tratados endodonticamente é um desafio que pode ser satisfatoriamente solucionado através da restauração endocrown. A constituição da técnica, baseada em uma coroa endodôntica adesiva unitária, não necessita de pinos de retenção, e seu preparo consiste apenas em uma retenção central, que utiliza como fator principal a câmara pulpar e o sistema adesivo. Objetivo: $O$ objetivo do presente trabalho foi apresentar um caso clínico por meio de uma restauração endocrown. Materiais e métodos: O caso clínico foi executado na Clínica Odontológica da Universidade Regional de Gurupi (UNIRG), em um indivíduo com dente posterior tratado endodonticamente com ampla destruição coronária. Conclusão: $O$ resultado obtido atendeu às exigências estéticas e funcionais esperadas.

Palavras-chave: Endocrown. Restauração adesiva. Pino endodôntico.

\section{ABSTRACT}

Introduction: The aesthetic and functional rehabilitation of teeth with extensive coronary destruction treated endodontically is a challenge that can be satisfactorily solved through endocrown restoration. The constitution of the technique, based on a unitary adhesive endodontic crown, does not require retention pins, and its preparation consists only of a central retention, which uses as its main factor the pulp chamber and adhesive system. Objective: The goal of this study was to present a case report of an endocrown restoration. Materials and methods: This case was executed at Dental Clinic University of Gurupi, in an individual whose posterior tooth had an extensive corona dentis destruction and was treated endodontically. Conclusion: The result obtained met the expected aesthetic and functional requirements.

Key-words: Endocrown. Adhesive restoration. Endodontic post. 


\section{INTRODUÇÃO}

A história da odontologia é caracterizada por diversos avanços científicos e tecnológicos, desse modo, o advento da intervenção minimamente invasiva e do sistema adesivo é um preceito indispensável na reabilitação oral, sendo necessário o desdobramento de técnicas e materiais capazes de proporcionar a resistência dentária e a retenção do material restaurador unido à cavidade. Nesse âmbito, dentes tratados endodonticamente com extensa destruição coronária são uma controversa por apresentarem grandes riscos de fratura devido à perda de substâncias do elemento dental. Assim, existindo uma discussão em relação à técnica adequada para a restauração destes dentes. $^{1}$

Antigamente, só se utilizava o protocolo de fabricação de coroas totais sustentadas por núcleos metálico-fundidos e/ou pinos de fibra de vibro; no entanto, apesar de promover retenção para a peça protética, acredita-se que a resistência do elemento dentário fique comprometida. Desse modo, a restauração endocrown se mostra eficaz na reabilitação estética e funcional. A literatura relata que molares restaurados com endocrowns transferem menos estresse funcional à dentina comparado com os molares restaurados com pinos e núcleos. ${ }^{2}$

A restauração endocrown constitui-se de uma coroa endodôntica adesiva unitária, que não necessita de pinos de retenção, minimizando a fragilização do conduto por meio de uma menor remoção da dentina, e por pouca ou nenhuma necessidade de desobturação, que faz com que não haja risco de contaminação do canal tratado. ${ }^{3}$

O preparo para endocrown consiste em uma retenção central que utiliza como fator principal a câmara pulpar e o sistema adesivo, com margem cervical circundante em esmalte, obtendo-se macro-retenção e micro-retenção. Dessa forma, essa técnica diminui o número de interfaces múltiplas adesivas, tornando a restauração menos suscetível aos efeitos adversos da degradação da camada híbrida. ${ }^{4}$

As endocrowns contemporâneas fabricadas com cerâmicas reforçadas, possuem excelente propriedade retentiva por serem ácido sensíveis (dissilicato de lítio), além do mais apresentam resistência considerável para cargas oclusais em dentes posteriores. Em vista disso, a utilização dessa cerâmica para este tratamento é relevante, uma vez que grandes cargas mastigatórias serão acometidas sobre a coroa dentária. ${ }^{5}$

A presença de núcleos pode causar forças oblíquas e horizontais, todavia, as endocrowns eliminam esses fatores, melhorando a distribuição de estresse nas superfícies 
dentárias, além de fornecerem menor custo e tempo clínico. Esta alternativa de tratamento, que propõe a máxima preservação da estrutura dental é indicada em casos de coroas curtas, espaço interoclusal limitado e/ou canais radiculares atrésicos, curvos, ou calcificados, que contraindicariam o uso de retentores intraradiculares. ${ }^{6}$ Nesse contexto, para obter a sobrevida clínica de uma restauração endocrown, o manejo adequado da técnica adesiva é primordial.

A restauração de dentes posteriores tratados endodonticamente é um desafio, diante disso, a endocrown viabiliza a preservação das estruturas dentárias com intervenção mais conservadora, se tornando uma aliada na longevidade da restauração diante de forças mastigatórias. Nessa perspectiva, o objetivo do presente trabalho foi apresentar um caso clínico por meio de uma restauração endocrown, enfatizando a definição, as indicações, vantagens, material utilizado e cimentação adesiva, ratificando a resistência mecânica e a função almejada.

\section{RELATO DE CASO}

O caso clínico foi submetido e aprovado pelo Comitê de Ética em Pesquisa da Universidade de Gurupi conforme parecer ํㅜ 3.711.155. Tratou-se de paciente de 72 anos de idade, gênero masculino, buscou a Clínica Escola de Odontologia da Universidade de Gurupi para uma reabilitação protética do elemento 36 desvitalizado com extensa destruição coronária. Após análise radiográfica para confirmação da adequada obturação endodôntica, e ao exame clínico do remanescente dentário, observou-se que havia espaço interoclusal limitado. Foi proposta reabilitação em três sessões mediante uma coroa endocrown, visto que o elemento dentário cumpria as corretas indicações (Figuras 1 e 2).

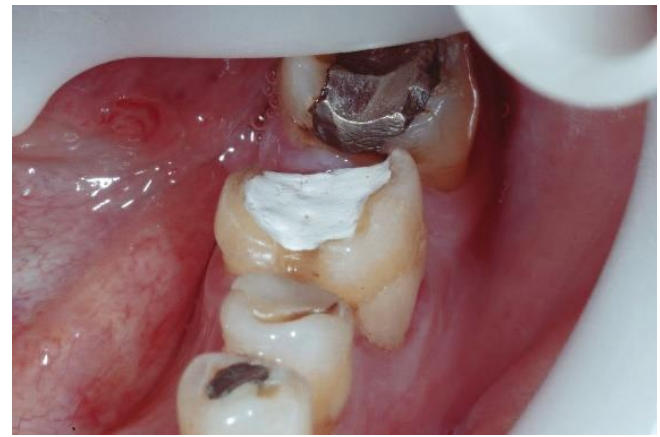

Figura 1. Aspecto clínico inicial do elemento 36.

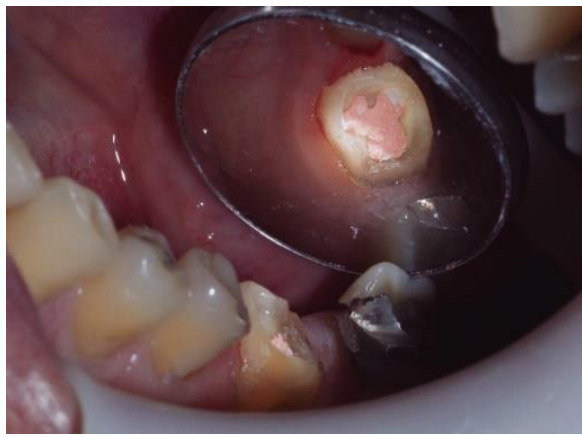

Figura 2. Elemento 36 após remoção da restauração provisória

Posteriormente ao consentimento para o tratamento, iniciou-se o preparo do elemento com o uso das pontas diamantadas 1014, 2200, 2131, 3131 e 3131F, buscando a remoção 
do material restaurador provisório e da estrutura coronal friável e potencializando a retenção da câmara pulpar, seguindo os princípios descritos na literatura, mantendo as paredes circundantes expulsivas, margens nítidas com término supragengival e em angulação de aproximadamente 90ㅜ, ângulos internos arredondados e redução de 2 a $3 \mathrm{~mm}$ de cúspides (Figura 3).

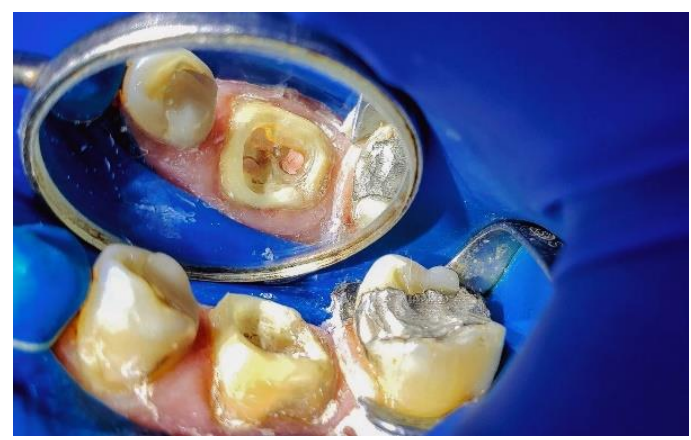

Figura 3. Elemento 36 após o preparo.

Após o preparo confeccionou-se um elemento provisório de resina acrílica (VIPIFLASH; DENCOR) a partir de uma escultura diretamente na boca e cimentado com um cimento a base de hidróxido de cálcio (HydroC) (Figuras 4 a 6).

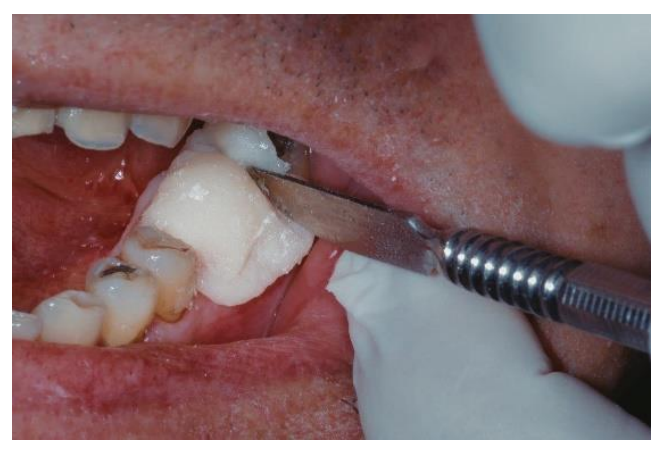

Figura 4. Confecção do elemento provisório.

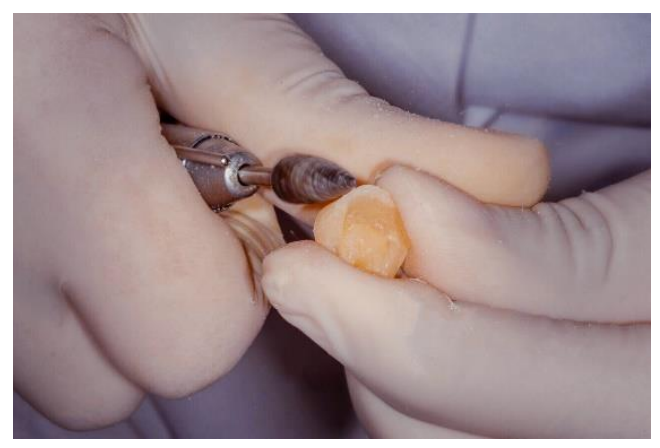

Figura 5. Acabamento do elemento provisório.

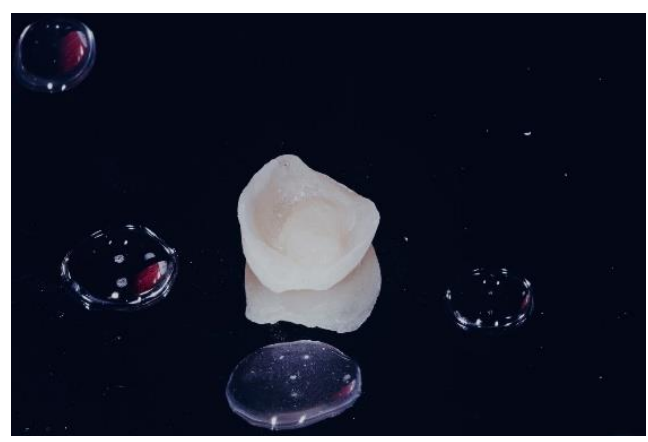

Figura 6. Elemento provisório de resina acrílica, vista interna.

Em sessão subsequente, para a etapa de moldagem, primeiramente foram inseridos fios de afastamento gengival a fim de facilitar a cópia do término cervical, usou-se o fio ultra 
extra fino (PRO RETRACT 000, FGM). O material de moldagem selecionado foi silicona de adição (FUTURA AD, DFL) pela técnica de tempo único. Inicialmente manipulou-se a pasta pesada inserindo-a na moldeira, em seguida, a silicona leve foi injetada através de uma pistola no preparo dental e sobre a pasta pesada. Após a remoção do fio retrator a moldeira foi inserida na boca do paciente, certificando-se de sua adaptação perfeita, e aguardou-se o tempo de polimerização (Figura 7). Por fim, foi realizada a moldagem da arcada antagonista com alginato (Hydrogum 5 - Zhermack).

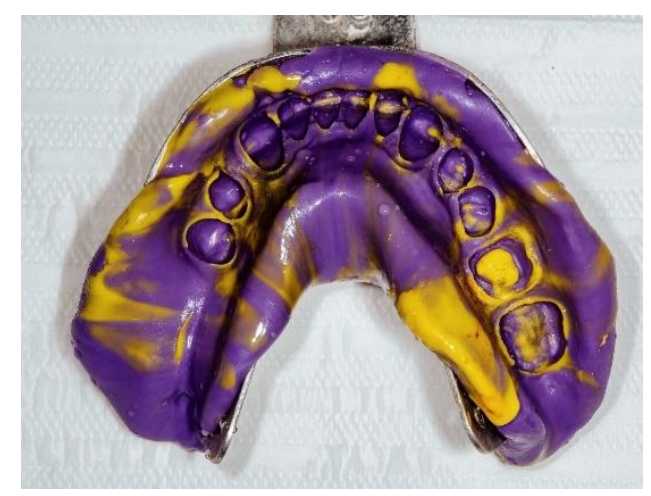

Figura 7. Molde da mandíbula com silicone de adição.

Na seleção da cor foi usada a escala de cor VITAM 3D-MASTER 3R, essa foi enviada, juntamente com o modelo de trabalho e o modelo do antagonista, ao laboratório de prótese, para confecção da peça pelo sistema cerâmico da técnica injetada (IPS E.Max).

$\mathrm{Na}$ consulta seguinte, foi realizada a prova da peça, analisando adaptação, pontos de contato interproximais e oclusais, cor e forma. Aprovados esses itens, iniciou-se o processo de cimentação. Realizou-se profilaxia do elemento dentário com pedra pomes (Pomitec) para remoção de resíduos do cimento provisório. A superfície interna da peça foi sequencialmente condicionada com aplicação de ácido fluorídrico a 10\% (CONDAC, FGM) por 20 segundos, seguida de abundante lavagem, e com ácido fosfórico a 37\% (CONDAC 37, FGM) por 30 segundos para a limpeza da peça. Logo após, passou-se uma fina camada de agente de união silano (PROSIL, FGM) aguardando por um minuto. O sistema adesivo (ESPE, 3M) foi aplicado e fotopolimerizado. Concomitante ao tratamento da peça, foi realizado o isolamento absoluto modificado do elemento, seu condicionamento com ácido fosfórico a 37\% (CONDAC 37, FGM) por 30 segundos em esmalte e 15 segundos em dentina, seguido de lavagem por igual tempo, secagem com papel absorvente e aplicação do mesmo sistema adesivo usado na peça. Foi utilizado o cimento resinoso adesivo (ALLCEM DUAL, FGM) passado em toda a extensão interna da peça e então assentada no elemento preparado, houve a fotopolimerização inicial, seguida da remoção dos excessos 
e a fotoativação em cada face da coroa que, somada a presa química, garantiu adequada cimentação. O isolamento foi removido e os contatos checados mediante o uso de carbono, havendo necessidade de ajuste oclusal, realizou-se tomada radiográfica final (Figuras 8 a 20). Posteriormente, foram realizadas sessão de proservação, nas quais observou-se sucesso clínico.

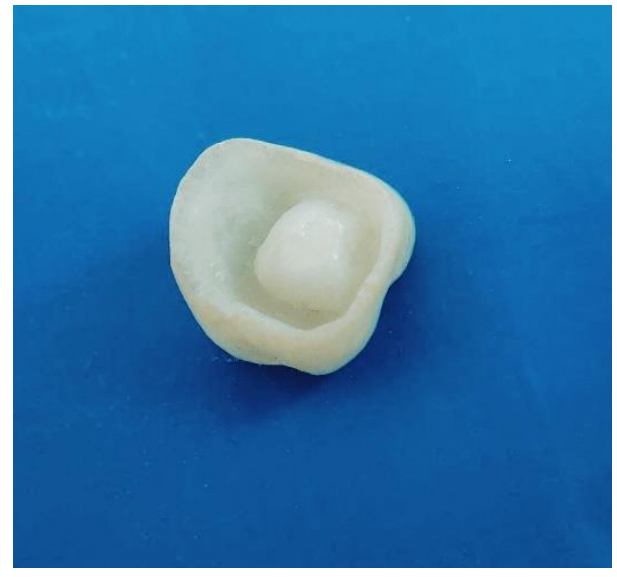

Figura 8. Endocrown de porcelana elemento 36, vista interna.

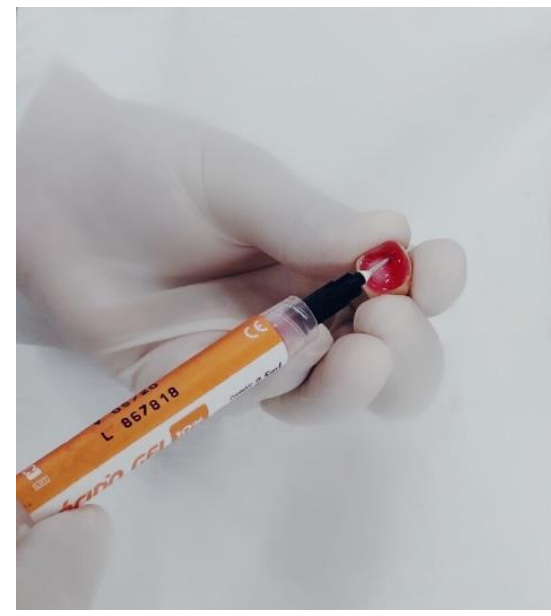

Figura 10. Ácido. Fluorídrico a 10\% para o condicionamento da cerâmica. Figura 11. Aplicação Ácido. Fosfórico a 37\% para limpeza.

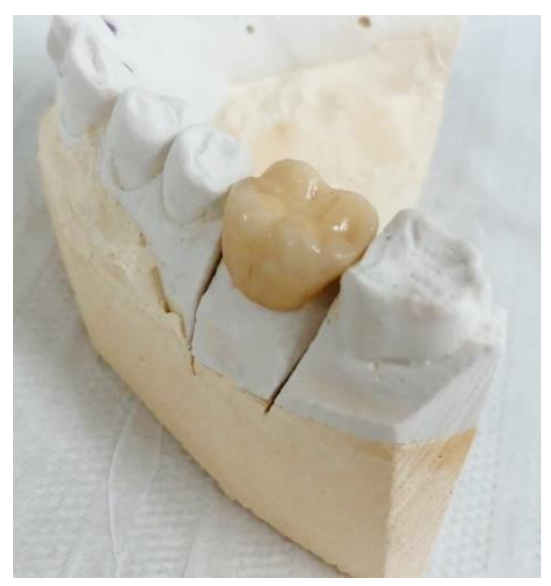

Figura 9. Endocrown porcelana elemento 36, vista externa.

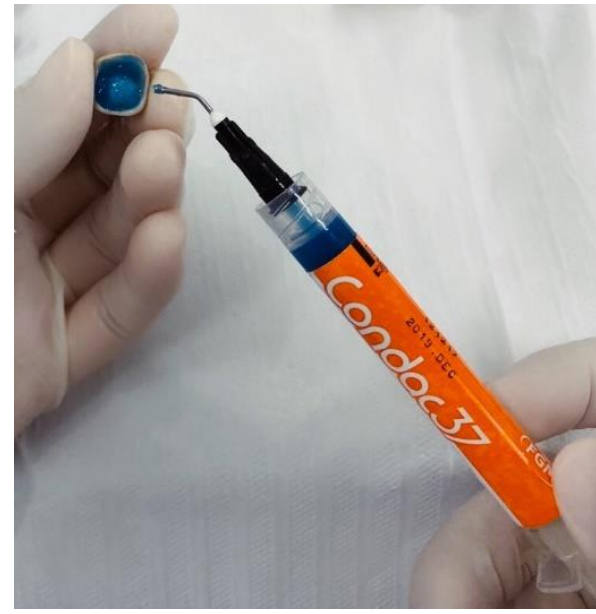


DOI: 10.18606/2318-1419/amazonia.sci.health.v8n3p50-60

Revista Amazônia Science \& Health

ISSN: 2318-1419

2020, Vol. 8 , №3

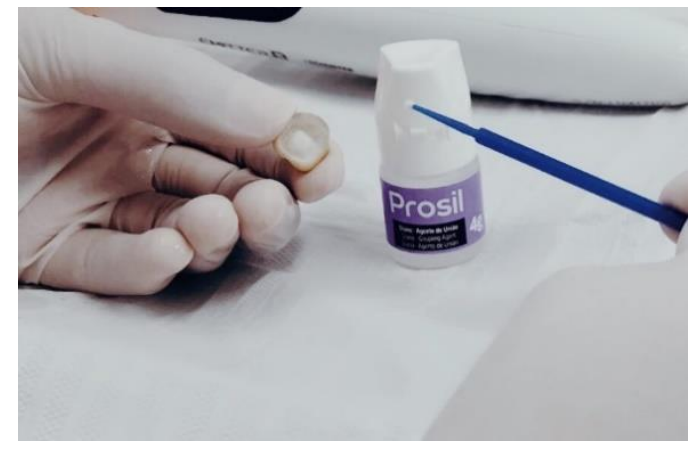

Figura 12. Aplicação do agente de união Silano na peça

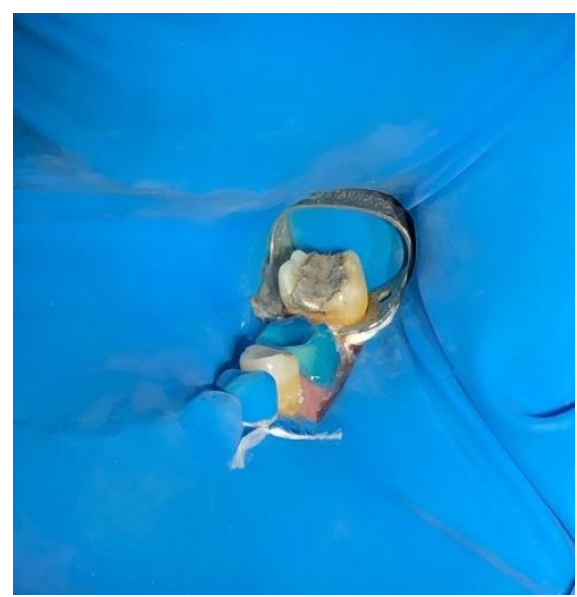

Figura 14. Ác. Fosfórico a 37\% no elemento 36.

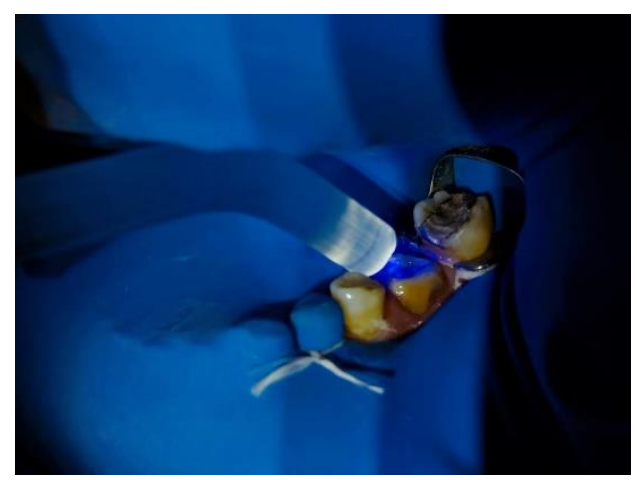

Figura 16. Fotopolimerização do adesivo aplicado no dente.
ÁVILA ALA, RAMOS VA, TORRES SMP.

Restauração Indireta Endocrown: Relato de Caso Clínico.

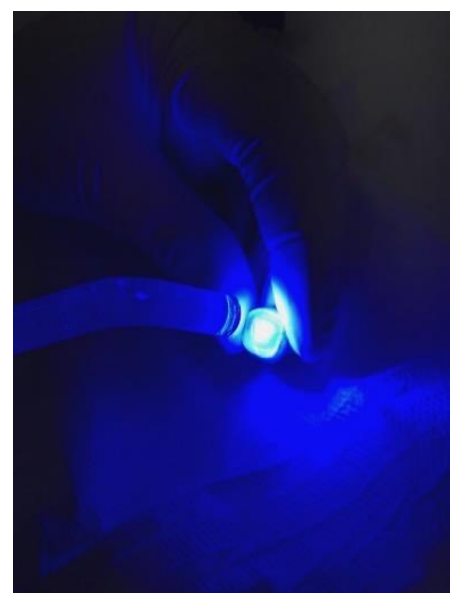

Figura 13. Fotopolimerização do sistema adesivo aplicada na peça.

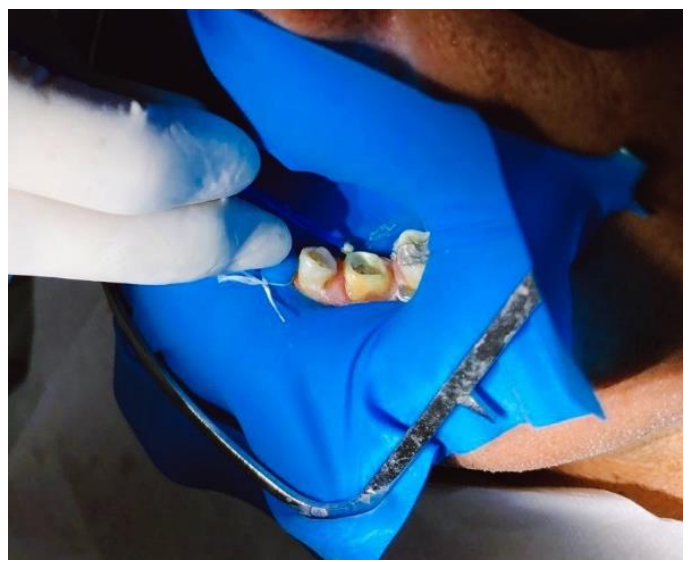

Figura 15. Aplicação do sistema adesivo no elemento 36.

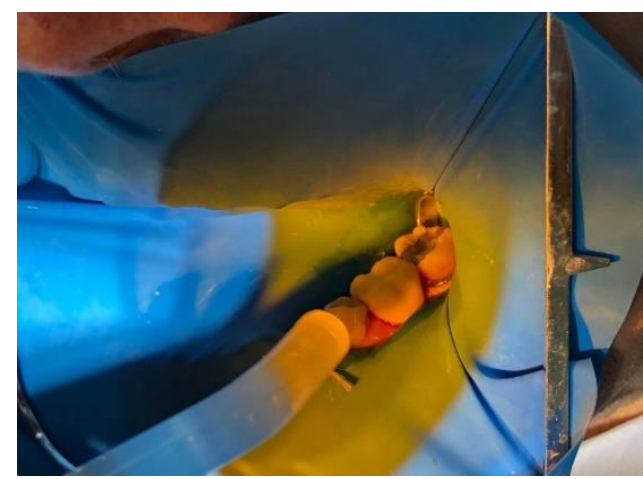

Figura 17. Assentamento da peça e fotopolimerização inicial. 


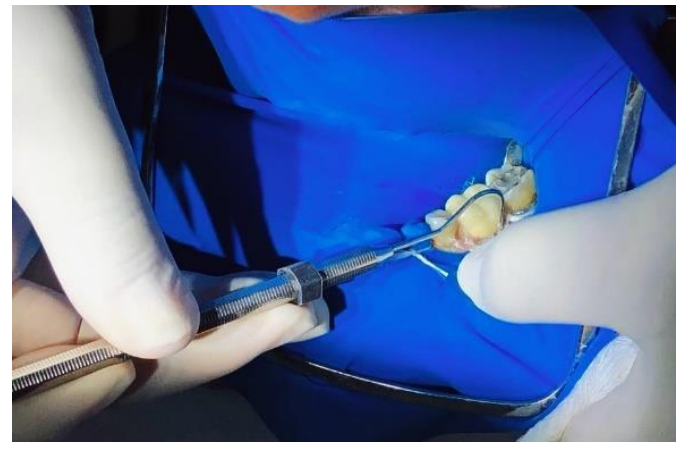

Figura 18. Remoção de excessos do cimento extravasado.

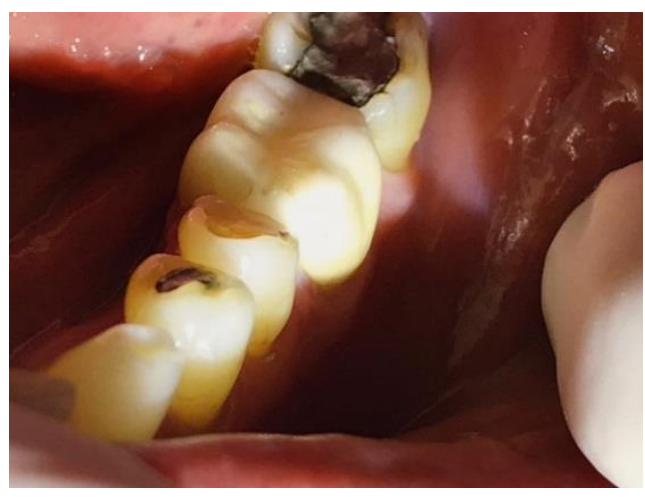

Figura 19. Endocrown elemento 36 após cimentação.

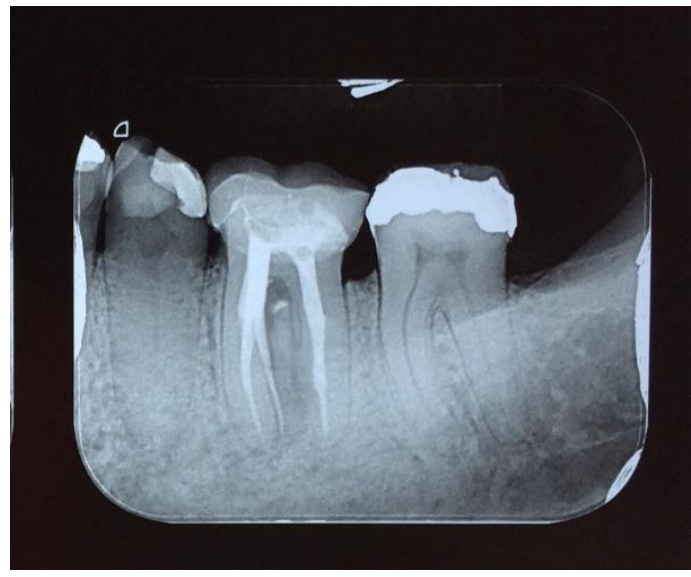

Figura 20. Radiografia periapical após cimentação.

\section{DISCUSSÃO}

Endocrowns têm sido uma alternativa promissora de tratamento para os molares tratados endodonticamente com coroas clínicas curtas, raízes obliteradas e paredes axiais finas. ${ }^{7}$ Menezes-Silva et al. em seu estudo citaram que a endocrown é uma técnica adequada para casos em que há grande perda de estrutura dentária, pequeno espaço interproximal em que a tradicional reabilitação com pino e coroa não é possível por causa da espessura inadequada da cerâmica. ${ }^{8}$ Carvalho et al. corroboram com os mesmos achados, onde avaliaram estudos com endocrowns e concluíram que estas executam de forma semelhante ou melhor do que os tratamentos convencionais usando pinos intraradiculares, resina composta direta ou restaurações inlay/onlay. ${ }^{9}$

Por meio de estudos in vitro que a força de fratura é significativamente superior para endocrowns do que coroas convencionais restauradas com um pino e núcleo ou com um pino de fibra e um núcleo de resina. Além disso, para melhores propriedades estéticas, resistência e adesividade, essas restaurações devem ser feitas apenas com cerâmica reforçada. ${ }^{7}$ Além do tipo de retentor, a friabilidade dos dentes despolpados, a variável 
anatomia das raízes e o risco de uma possível perfuração radicular durante o preparo, tornam ainda mais favorável a utilização da técnica endocrown. ${ }^{8}$

Biacchi et al. realizaram um estudo, em que o grupo com restaurações endocrown obteve resultados melhores na resistência à compressão, quando comparado com os molares restaurados com pino de fibra de vidro. ${ }^{4}$

A restauração endocrown em molares é mais benéfica do que em pré-molares, devido à grande superfície da câmara pulpar, o que melhorará a retenção micromecânica fornecida pelo sistema adesivo. Assim, as endocrowns devem se limitar à restauração de molares tratados endodonticamente, a fim de obter um desempenho satisfatório em termos de resistência de união e resposta a cargas oclusais. ${ }^{10}$ Molares desvitalizados restaurados com pinos de fibra e coroas cerâmicas, são mais propensos à falhas do que molares restaurados com endocrowns. ${ }^{2,11}$

Para a fabricação da endocrown, de cerâmica monolítica à base de dissilicato de lítio, IPS e.max (Ivoclar Vivadent, Schaan, Liechtenstein), possui adequadas propriedades físicas e maior translucidez. De acordo com o fabricante, existem dois tipos de cerâmica do sistema IPS e.max: o tipo monolítico com uma dimensão oclusal de 1,5 mm de espessura, sem a necessidade de revestimento de porcelana; e o dissilicato de lítio coping (mínimo 0,8 $\mathrm{mm}$ ) coberto com um revestimento de porcelana (máximo 0,7 $\mathrm{mm}$ ). Ambos têm força suficiente para a restauração de dentes posteriores, além de não promoverem desgaste excessivo do dente antagonista. ${ }^{8}$

Em contrapartida, outros estudos concluíram que as cerâmicas são consideradas materiais de alta rigidez com alto módulo de elasticidade (210 GPa). Materiais de alta rigidez, como alumina, podem resultar em carga excessiva porque a transmissão de força oclusal pode ter um impacto negativo nas propriedades biomecânicas da restauração e dos dentes pilares. Zoidis demonstrou que materiais com um módulo de elasticidade menor, como o Poliéter-éter-cetona (PEEK) e as resinas compostas provaram reduzir o estresse oclusal, agindo como amortecedores. PEEK exibe um módulo de elasticidade de $4 \mathrm{GPa}$, o que poderia amortecer a transmissão da força, impedindo assim a sobrecarga e quebra do dente e subseqüentemente da raiz. Além disso, o PEEK como material principal reduz a elasticidade do revestimento de resina composta, material de 8 a $10 \mathrm{GPa}$ a $4 \mathrm{Gpa}^{7}$

Nessa perspectiva, Tribst et al., demonstraram que quanto maior o módulo de elasticidade do material, maior a concentração de tensão na restauração e menor a concentração de tensão na linha de cimento; e que quanto maior o remanescente da coroa 
dentária, maior a concentração de estresse na restauração. Assim, o restante tecido dentário deve ser sempre preservado. ${ }^{12}$

Menezes-Silva et al., justificaram a opção pelo uso de cimento dual pelo fato que a unidade de fotopolimerização de luz necessita passar através da espessura da cerâmica. AllCem é um cimento adesivo permanente, de dupla polimerização, é radiopaco e tem uma largo espectro de aplicação em restaurações indiretas. A combinação de dois mecanismo de polimerização físicos e químicos, garantem polimerização do produto em situações mesmo na ausência de acesso à luz. Tem adequada propriedades de adesão química, e também é fácil de aplicar devido à seringa com corpo duplo que auto mistura o cimento e impede a incorporação de bolhas de ar. A pré-polimerização do cimento permite a remoção de excessos mais facilmente, sem a necessidade de retirada dos restos mecânicos do cimento que pode ter resultado em trauma ao tecido gengival marginal, o que pode levar a recessão gengival indesejável. ${ }^{8}$

\section{CONCLUSÁO}

As coroas endodônticas adesivas - Endocrowns representam uma alternativa de tratamento muito esperançosa para os molares tratados endodonticamente, pois permitem a manutenção da estrutura dentária, são compatíveis com a odontologia minimamente invasiva e adequadas para o conceito de biointegração. Nessa perspectiva, conclui-se que a técnica aborda todos os pontos necessários para se adquirir uma restauração indireta adequada para dentes posteriores. O estudo de caso apresentou estética semelhante ao dente natural, função e resistência à forças oclusais, obtendo-se o desfecho almejado.

Esse tipo de reconstrução, que ainda é incomum, deve ser mais conhecido e praticado, em vista que ao seguir rigorosamente as corretas indicações a técnica apresenta poucas intercorrências.

\section{REFERÉNCIAS}

1 Dogui H, Abdelmalek F, Amor A, Douki N. Endocrown: An Alternative Approach for Restoring Endodontically Treated Molars with Large Coronal Destruction. Case reports in dentistry. 2018.

2 Hayes A, Duvall N, Wajdowicz M, Roberts H. Effect of endocrown pulp chamber extension depth on molar fracture resistance. Operative dentistry. 2017;42(3):327-334. 
3 Hamdy A. Effect of full coverage, endocrowns, onlays, inlays restorations on fracture resistance of endodontically treated molars. Journal of Dental and Oral Health. 2015;5:2.

4 Biacchi GR, Mello B, Basting RT. The endocrown: an alternative approach for restoring extensively damaged molars. Journal of Esthetic and Restorative Dentistry. 2013;25 (6):383-390.

5 Baccarin AN, Zaze CA. Coroa endodôntica adesiva: relato de caso clínico. Rev. Odontol. 2012; 33(2):47-51.

6 Hasan I, Frentzen M, Utz KH, Hoyer D, Langenbach A, Bourauel C. Finite element analysis of adhesive endo-crowns of molars at different height levels of buccally applied load. Journal of dental biomechanics. 2012;3.

7 Zoidis P, Bakiri E, Polyzois G. Using modified polyetheretherketone (PEEK) as an alternative material for endocrown restorations: A short-term clinical report. The Journal of prosthetic dentistry. 2017;17(3):335-339.

8 Menezes-Silva R, Espinoza CAV, Atta MT, Navarro MFL, Ishikiriama SK, Mondelli RFL. Endocrown: a conservative approach. Brazilian Dental Science. 2016;19(2):121-131.

9 Carvalho MAD, Lazari PC, Gresnigt M, Del Bel Cury AA., Magne P. Current options concerning the endodontically-treated teeth restoration with the adhesive approach. Brazilian oral research.2018;32.

10 Helal MA, Wang Z. Biomechanical Assessment of Restored Mandibular Molar by Endocrown in Comparison to a Glass Fiber Post-Retained Conventional Crown: 3D Finite Element Analysis. Journal of Prosthodontics. 2017.

11 Dejak B, Młotkowski A. 3D-Finite element analysis of molars restored with endocrowns and posts during masticatory simulation. Dental Materials. 2013;29(12):309-317.

12 Tribst JPM, Piva AMOD, Madruga CFL, Valera MC, Borges ALS, Bresciani E, et al. Endocrown restorations: Influence of dental remnant and restorative material on stress distribution. Dental Materials. 2018;34(10):1466-1473. 